\title{
Diabetic Foot: Infections and Outcomes in Iranian Admitted Patients
}

\author{
Azar Hadadi ${ }^{1, *}$; Houra Omdeh Ghiasi ${ }^{2}$; Mahboubeh Hajiabdolbaghi ${ }^{3}$; Majid Zandekarimi ${ }^{4}$; \\ Reza Hamidian $^{5}$ \\ ${ }^{1}$ Department of Internal Medicine, Research Development Center, Sina Hospital, Tehran University of Medical Sciences, Tehran, IR Iran \\ ${ }^{2}$ Sina Hospital, Tehran University of Medical Sciences, Tehran, IR Iran \\ 3 Department of Infectious Diseases, Iranian Research Center for HIV and AIDS, Emam Khomeini Hospital, Tehran University of Medical Sciences, Tehran, IR Iran \\ 4 Department of Infectious Diseases, Iranian Research
Zabol University of Pharmacology, Zabol, IR Iran \\ ${ }_{5}$ Zabol University of Pharmacology, Zabol, IR Iran \\ ${ }^{*}$ Corresponding author: Azar Hadadi, Department of Internal Medicine, Research Development Center, Sina Hospital, Tehran University of Medical Sciences, Sina Hospital, Imam \\ Khomeini St. Hasan Abad Square, Tehran, IR Iran. Tel/Fax:+98-2166348555, E-mail: hadadiaz@tums.ac.ir
}

Received: April 20, 2013; Revised: November 30, 2013; Accepted: February 11, 2014

\begin{abstract}
Background: Diabetes mellitus (along with its complications) has become a global problem. Diabetic foot infection, among the most common complications, is responsible for 40 to 50\% of foot amputations. Antibiotic-resistant microorganisms, however, have compromised empiric therapy in the infected patients.

Objectives: The current study aimed to determine the most common microorganisms involved in diabetic foot infection in order to minimize the failure of antibiotic therapy and the risk of developing complications.

Patients and Methods: All patients with diabetic foot infection admitted to the infectious diseases, surgery and endocrinology wards of two teaching hospitals from 2007 to $2010(n=196)$ were recruited. In this retrospective study, demographic characteristics, type of lesions, history of hospitalization/antibiotic therapy, isolated microorganisms, clinical complications, administered treatment (medical or surgical) and outcome were recorded.

Results: Patients' mean age was $60.84( \pm 10.30)$ years. Totally, $113(57.65 \%)$ of the patients were male and $83(42.35 \%)$ were female. According to Wagner's grading, deep ulcers with/without osteomyelitis accounted for the majority of lesions. A single microorganism was isolated (most common: Escherichia coli, Staphylococcus aureus and Klebsiella spp.) from 81 of the patients (80.20\%); while for the remaining polymicrobial infection was reported. Isolated pathogens showed no significant correlation with duration of diabetes, type of the lesions $(\mathrm{P}=0.13)$ and history of hospitalization $(\mathrm{P}=0.61)$. The majority of patients $(\mathrm{n}=118,60.20 \%)$ were treated surgically; however 11 patients expired due to sepsis. Amputation (most common at toes and below the knee) was performed for 89 patients (45.40\%). The response rate to medical treatment was $31.6 \%$ for single-pathogen and $10 \%$ for polymicrobial infection (with a 30\% mortality rate).

Conclusions: Physicians are recommended to take microbiological cultures before starting empirical therapy recommended to cover Gram-negative microorganisms in order to lower the risk of antibiotic resistance.
\end{abstract}

Keywords:Diabetic Foot; Infection; Inpatients; Outcome Assessment

\section{Background}

As a globally widespread disease with an increasing incidence, diabetes mellitus has afflicted 150,000,000 people across the world according to the World Health Organization (WHO); and this will be doubling by 2025, (1). Not only the diabetes itself, but also its complications impose a heavy health and economic burden on the societies and health systems. Infections and ulcers accompanied by neuropathy and arteriovenous abnormalities in the foot of patients with diabetes, referred as diabetic foot, are among the most common complications, $(2,3)$. Diabetic foot infection, also considered as the most important cause of hospitalization in patients with diabetes accounted for 20\% of inpatient admissions in North America is responsible for a large number of foot amputations worldwide, $(3,4)$. Recent studies have estimated that more than 1.5 million Iranians have diabetes, and 7.5\% of them are diagnosed with diabetes mellitus type 2, (5); thus diabetic foot infection is a common problem in Iran as well.

Different microorganisms are isolated from diabetic foot infections, based on severity and depth of ulcers. For instance, Gram-positive cocci are the most common germs in superficial ulcers, while anaerobic bacteria are mostly found in deeper lesions (4). The presence of different microorganisms along with increasing resistance to antibiotic therapy has compromised the empiric therapy in diabetic foot infection.

\section{Objectives}

The current study aimed to determine the most common microorganisms responsible for diabetic foot infections in order to minimize the failure of antibiotic therapy and the risk of developing complications (including amputation) in a group of Iranians. 
Hadadi A et al.

\begin{tabular}{|c|c|c|}
\hline Wagner's Grade & Signs & No. (\%) \\
\hline $\mathbf{0}$ & No ulcer in a high risk foot & $0(0.0)$ \\
\hline 1 & Superficial ulcer involving the full skin thickness & $23(11.79)$ \\
\hline 2 & Deep ulcer penetrating to ligaments/muscle, but no bone involvement or abscess formation & $56(28.71)$ \\
\hline 3 & Deep ulcer with cellulitis or abscess formation, often with osteomyelitis & $93(4769)$ \\
\hline 4 & Localized gangrene & $23(1179)$ \\
\hline 5 & Extensive gangrene involving the whole foot & $10(5.12)$ \\
\hline
\end{tabular}

Table 2. Frequency of Microorganisms Isolated From 119 Microbiological Cultures of Diabetic Foot Infection

\begin{tabular}{lc}
\hline Microorganism & No.(\%) \\
\hline E. coli & $34(28.57)$ \\
\hline Staphylococcus aureus & $25(21.00)$ \\
\hline Klebsiella spp. & $17(14.28)$ \\
\hline Pseudomonas spp. & $10(8.40)$ \\
\hline Acinetobacter spp. & $5(4.20)$ \\
\hline Proteus spp. & $5(4.20)$ \\
\hline Streptococcus species & $5(4.20)$ \\
\hline Citrobacter spp. & $4(3.36)$ \\
\hline Stenotrophomonasmaltophilia & $4(3.36)$ \\
\hline Enterococcus spp. & $4(3.36)$ \\
\hline Edwardisiella spp. & $3(2.52)$ \\
\hline Providencia spp. & $3(2.52)$ \\
\hline Total & $119(100)$ \\
\hline
\end{tabular}

\section{Patients and Methods}

This retrospective cross-sectional multicenter study was conducted in two teaching hospitals affiliated to Tehran University of Medical Sciences, Tehran, Iran. All patients with diabetic foot admitted to the infectious diseases, surgery and endocrinology wards of these two hospitals from 2007 to 2010 were recruited. The diagnosis was made based on WHO criteria for diabetes mellitus diagnosis (approved by American Diabetes Association), (5). The Ethics Board Committee of Tehran University of Medical Sciences approved the study, in conformity to the Declaration of Helsinki. The committee also waived the need for "informed consent" because all subjects had declared the permission for using their data on condition of anonymity at admission.

The patients' demographic characteristics, type of diabetic foot lesions, history of hospitalization, history of antibiotherapy, frequency of isolated microorganisms (according to microbiological cultures), clinical complications, administered treatment (medical or surgical), and relevant outcome were extracted from medical records. The surgical procedures mainly adopted in these patients included abscess drainage, debridement and amputation.
The specimens were checked for aerobic and anaerobic microorganisms through streak culturing them on Eosin methylene blue (EMB), Blood Agar (BA) and chocolate Agar (CA) (Asan Pharmaceutical Co. Ltd. South Korea). Plates were incubated overnight at an incubation temperature of $37^{\circ} \mathrm{C}$. In case any bacteria grew on the plates, the specimens would be screened by Gram stain for the presence of multiple types of organisms. Then, the antibiogram was conducted on Muller Hinton Agar using the CLSI disk-diffusion method.

\subsection{Statistical Analysis}

The frequency of characteristics was presented as number (percentage), and quantitative results as mean ( \pm standard deviation). Chi-square test was applied to compare qualitative characteristics and Student t-test was applied for quantitative ones; the quantitative characteristics that did not have normal distribution, however, were compared with Mann-Whitney test. Response to treatment was determined by means of logistic regression analysis. All statistical calculations were done with SPSS ver. 17 (IBM, USA). P value $<0.05$ was considered significant.

\section{Results}

One hundred and ninety six patients with the mean age of $60.84( \pm 10.30)$ years were recruited. They included 113 male (57.65\%) and 83 female (42.34\%), and among them, 34 patients $(17.34 \%)$ had type I and 162 patients $(82.65 \%)$ lived with type II diabetes. The mean duration of diabetes mellitus and diabetic foot ulcers were $18.11( \pm 10.03)$ years and 48.98 ( \pm 63.60$)$ days respectively. Fifty-six patients (28.57\%) had already received antibiotics before admission. Previous history of hospitalization was reported in 47 patients (23.98\%). Information about types of lesions according to Wagner's grading is summarized in Table 1.

Table 2 outlines the results of 119 microbiological cultures (51.53\%). A single microorganism was isolated from 81 patients (80.20\%), whereas the other 20 patients (19.8\%) were diagnosed with polymicrobial infection. Escherichia coli, Staphylococcus aureus and Klebsiella spp. formed the most common pathogens found in the cultures. Taking type of lesion into account, S. aureus, Streptococcus species, and E. coli were the most frequent microorganisms in abscess, and S. aureus, Streptococcus species, and Klebsiella spp. were more common in oozing ulcers. 
Hadadi A et al.

Table 3. The Outcomes of Medical/Surgical Treatment of Diabetic Foot Infection According to the Microbiological Cultures at the Time of Hospital Discharge ${ }^{a, b}$

\begin{tabular}{lccccc}
\hline Culture & $\begin{array}{c}\text { Treatment } \\
\text { Failure }\end{array}$ & $\begin{array}{c}\text { Death Due } \\
\text { to MI }\end{array}$ & $\begin{array}{c}\text { Death Due to } \\
\text { Sepsis }\end{array}$ & $\begin{array}{c}\text { Recovery After } \\
\text { Surgery }\end{array}$ & $\begin{array}{c}\text { Recovery After Medical } \\
\text { Therapy }\end{array}$ \\
\hline Positive $(\mathbf{n}=\mathbf{1 0 1})$ & $9(8.91)$ & $2(1.98)$ & $7(69.30)$ & $60(59.40)$ & $23(22.77)$ \\
Negative $(\mathbf{n}=\mathbf{9 5})$ & $20(21.05)$ & $0(0.00)$ & $2(2.10)$ & $34(35.79)$ & $39(41.05)$ \\
Total sum $(\mathbf{n}=\mathbf{1 9 6})$ & $29(14.79)$ & $2(1.02)$ & $9(4.59)$ & $94(47.96)$ & $62(31.63)$ \\
\hline
\end{tabular}

a Abbreviation: MI, myocardial infarction.

b Values are shown as No.(\%).

Isolated pathogens showed no significant correlation with duration of diabetes $(\mathrm{P}=0.13)$, duration of foot lesions $(\mathrm{P}=0.13)$, type of lesions $(\mathrm{P}=0.20)$ and history of hospitalization for diabetic food infection $(P=0.61)$. Similarly, the relationship of single or polymicrobial infection with type of lesions $(\mathrm{P}=0.10)$ and history of hospitalization for diabetic food infection ( $\mathrm{P}=1.00)$ was insignificant, on the contrary, the association between isolated pathogen and patients' outcome at the time of discharge was statistically significant $(\mathrm{P}<0.001)$. The polymicrobial infections led to worse outcome at the time of discharge $(\mathrm{P}<0.001)$.

The majority of patients $(n=118,60.20 \%)$ were treated surgically and only 78 patients (39.80\%) benefited from mere antibiotic therapy. Amputation was performed for 89 patients (45.40\%). The level of amputation ranged from toes $(n=47)$ to below the knee $(n=36)$, distal of metatarsus ( $n$ $=9)$ and above knee $(n=8)$. In the meantime, 25 patients (12.1\%) benefitted from debridement. The response rate to medical treatment was $31.6 \%$ for single pathogen infection vs.10\% for polymicrobial infection. A 30\% mortality rate was observed in the latter group. Eleven patients also expired due to sepsis. Table 3 outlines the outcome of treatment at the time of hospital discharge. This table is adjusted according to patients' microbiological cultures; therefore, the numbers in categories are less than the relevant total number of patients. Treatment failure was calculated as a total of lack of response to antibiotherapy and/or surgical intervention plus discharge against medical advice.

\section{Discussion}

Diabetic foot infection spans from local to necrotizing and life-threatening infections between malleoli and toes, (6). It is the second leading cause of lower limb amputation in North America, (4). Although the prevalence of diabetes type II is reported $7 \%$ to $8 \%$ for major cities of Iran, Southern Iran is reported to have remarkably higher rate (up to 17\%). Recent estimates show that diabetic foot infections occur in 3\% of the Iranian population, (3), indicating that approximately 225,000 patients with diabetic foot complications impose a heavy burden on the health system in Iran. Published literature within the past decades have always considered $S$. aureus as the pathogen most likely found in diabetic foot lesions, (4, 7-13); while E. coli was the most frequent microorganism found in the present study.
A published report from Malaysia has also accorded with the same results, stating Gram-negative pathogens (including Proteus spp., Pseudomonas aeruginosa, Klebsiella pneumonia and E. coli) were the most common isolated microorganisms (52\%), (14). This comes when according to another study from Iran, E. coli stood as the second most common pathogen with minor discrepancy behind S. aureus (15). The prevalence of polymicrobial infections in this study was also remarkably higher than that of the current study (51\% vs. 19.8\%) (15). The majority of the current study patients had received empiric antibiotic therapy before admission; this may explain the differences noted in the isolated microorganism of the present study and the previous ones. On the contrary to current report, Pittet et al. reported that osteomyelitis, deep tissue infections, and gangrene as the most common lesions in patients with diabetic foot infection,(13). Morales Lozano et al. similarly reported osteomyelitis in $79.5 \%$ of their patients (16). The lower prevalence of osteomyelitis in the current study could be due to the fact that advanced diagnostic measures were not applied in the study and the diagnosis was made based on clinical findings. Alavi et al., however, reported a single microorganism, mainly S. aureus, as the most frequently isolated bacteria from diabetic foot patients (17).

The current study failed to report any relationship between the patient's outcome and demographic characteristics, history of hospitalization, duration of diabetes mellitus, neutrophil count and the anatomic site of foot lesions. This comes while in another study, the complication of treatment of diabetic foot infection depended on depth of ulcer, presence of ischemia and severity of glycemic control (18). The patients' age, gender, type and duration of diabetes and the anatomic site of ulcers did not correlate with the outcome (18). Similarly, Yekta et al. reported the association between the patients' characteristics and his/ her outcomes, stating that those with amputation were significantly older, - less educated, had longer duration of diabetes and had a poor glycemic control (3).

The association between BMI and amputation shown in other studies $(3,19,20)$, remained unproved in the current work. Correspondingly, while another study in Iran emphasized the correlation between patients' gender and amputation (21), the current study could not confirm such an association. Such a correlation, however, was not reported by Li et al. in their study on a population with a $21.8 \%$ rate of amputation in China (22). 
Amputation rate in the current study not only was higher than that of Yekta et al. (3) but also outweighed a decreasing rate of $40 \%$ to $12 \%$ by Larijani et al. who took a 22 year trend into account (23). As an implicit finding, slight complications were observed when more microbiological cultures were requested by the physician. Therefore, the treating physicians are recommended to send more samples in shorter time intervals to better diagnosis and choice of antibiotic.

\subsection{Limitations:}

Considering the fact that the data were extracted from the patients' medical records, missing some data was inevitable. It should be stressed that the results of the baseline culture of some patients were not available; this could explain some of the discrepancy noted between current study findings and those of previous researches. Moreover, since anaerobic culture was not accessible in the hospitals, anaerobic pathogens were not studied in diabetic foot lesions. The diagnosis of osteomyelitis in the current study was based on imaging reports rather than bone biopsy, which is a more definite diagnostic method.

The high rate of amputation noted in the current study could be contributed to the fact that the majority of the patients were referred to the centers late. Physicians are recommended to take microbiological cultures before starting empirical antibiotic therapy, which is recommended to cover Gram-negative microorganism, to lower the risk of experiencing antibiotic resistance. Educating the patients and asking them to visit the diabetic foot clinic more frequently could lower the complication rate in these patients.

\section{Acknowledgements}

The authors hereby thank Research Development Center of Sina Hospital, staff of Patients' Archive and Registry Department of Sina and Emem Khomeini Hospitals for their sincere supports and Dr. patricia khashayar for english editing.

\section{Authors' Contribution}

Study concept and design: Hadadi, Hajabdolbaghi and Omdeh Ghiasi. Analysis and interpretation of data: Hadadi, Hajabdolbaghi, Zandekarimi, Omdeh Ghiasi and Hamidian, Drafting of the manuscript: Hadadi and Hamidian. Critical revision of the manuscript for important intellectual content: Hadadi, Hajabdolbaghi, Zandekarimi, Omdeh Ghiasi and Hamidian. Statistical analysis: Hadadi, Zandekarimi, Omdeh Ghiasi and Hamidian.

\section{Financial Disclosure}

There is no financial interest.

\section{Funding/Support}

This study was supported and funded by Tehran University of Medical Sciences, Tehran, Iran.

\section{References}

1. World Health Organization . Diabetes Mellitus. Fact Sheet N 138 2012. Available from: http://www.who.int/mediacentre/factsheets/fs138/en.

2. Tesfaye S, Selvarajah D. Advances in the epidemiology, pathogenesis and management of diabetic peripheral neuropathy. Diabetes Metab Res Rev. 2012;28 Suppl 1:8-14.

3. Yekta Z, Pourali R, Nezhadrahim R, Ravanyar L, Ghasemi-Rad M. Clinical and behavioral factors associated with management outcome in hospitalized patients with diabetic foot ulcer. Diabetes Metab Syndr Obes. 2011;4:371-5.

4. Abdulrazak A, Bitar ZI, Al-Shamali AA, Mobasher LA. Bacteriological study of diabetic foot infections. J Diabetes Complications. 2005;19(3):138-41.

5. Larijani B, Zahedi F. Epidemiology of diabetes mellitus in Iran. Iran J Diabetes Lipid Dis. 2002;1(1):1-8.

6. Dalla Paola L, Faglia E. Treatment of diabetic foot ulcer: an overview strategies for clinical approach. Curr Diabetes Rev. 2006;2(4):431-47.

7. Espinosa Y, Nieves B, Quintana A. Aerobic and Anaerobic Bacteria in Diabetic Foot Disease. Anaerobe. 1999;5(3-4):405-7.

8. El-Tahawy AT. Bacteriology of diabetic foot. Saudi Med J. 2000;21(4):344-7.

9. Unachukwu $\mathrm{CN}$, Obunge OK, Odia OJ. The bacteriology of diabetic foot ulcers in Port Harcourt, Nigeria. Niger J Med. 2005;14(2):173-6.

10. Yoga R, Khairul A, Sunita K, Suresh C. Bacteriology of diabetic foot lesions. Med J Malaysia. 2006;61 Suppl A:14-6.

11. Lipsky BA, Berendt AR, Deery HG, Embil JM, Joseph WS, Karchme AW, et al. Diagnosis and treatment of diabetic foot infections. Plast Reconstr Surg. 2006;117(7 Suppl):212S-38S.

12. Beliaeva OA, Kryzhevskii W, Rybianets Iu V, Korzhik NP, Ulanovich LI, Shevchenko AV, et al. [The structure of microflora in ulcerative-necrotic lesions in patients with diabetic foot syndrome]. Klin Khir. 2010(10):20-3.

13. Pittet D, Wyssa B, Herter-Clavel C, Kursteiner K, Vaucher J, Lew PD. Outcome of diabetic foot infections treated conservatively: a retrospective cohort study with long-term follow-up. Arch Intern Med.1999;159(8):851-6.

14. Raja NS. Microbiology of diabetic foot infections in a teaching hospital in Malaysia: a retrospective study of 194 cases. J Microbio Immunol Infect. 2007;40(1):39-44.

15. Dezfulian A, Salehian MT, Amini V, Dabiri H, Azimi Rad M, Aslani $\mathrm{MM}$, et al. Bacteriological study of diabetic foot infections in an Iranian hospital. Iran Red Crescent Med J. 2011;13(8):590-1.

16. Morales Lozano R, Gonzalez Fernandez ML, Martinez Hernandez D, Beneit Montesinos JV, Guisado Jimenez S, Gonzalez Jurado MA. Validating the probe-to-bone test and other tests for diagnosing chronic osteomyelitis in the diabetic foot. Diabetes Care. 2010;33(10):2140-5

17. Alavi A, Sanjari M, Haghdoost A, Sibbald RG. Common foot examination features of 247 Iranian patients with diabetes. Int Wound J. 2009;6(2):117-22.

18. Oyibo SO, Jude EB, Tarawneh I, Nguyen HC, Armstrong DG, Harkless LB, et al. The effects of ulcer size and site, patient's age, sex and type and duration of diabetes on the outcome of diabetic foot ulcers. Diabet Med. 2001;18(2):133-8.

19. Yesil S, Akinci B, Yener S, Bayraktar F, Karabay O, Havitcioglu $\mathrm{H}$, et al. Predictors of amputation in diabetics with foot ulcer: Single center experience in a large Turkish cohort. Hormones. 2009;8(4):286-95.

20. Sohn MW, Budiman-Mak E, Lee TA, Oh E, Stuck RM. Significant J shaped association between body mass index (BMI) and diabetic foot ulcers. Diabetes Metab Res Rev. 2011;27(4):402-9.

21. Tabatabaei Malazy O, Mohajeri-Tehrani MR, Pajouhi M, Shojaei Fard A, Amini MR, Larijani B. Iranian Diabetic Foot Research Network. Adv Skin Wound Care. 2010;23(10):450-4.

22. Li X, Xiao T, Wang Y, Gu H, Liu Z, Jiang Y, et al. Incidence, risk factors for amputation among patients with diabetic foot ulcer in a Chinese tertiary hospital. Diabetes Res Clin Pract. 2011;93(1):26-30.

23. Larijani A, Afshari M, Darvish nezhad F, Bastan Hagh MH, Pazhouhi M, Baradar Jalili R, et al. Lower limb amputation in patients with diabetic foot ulcer: A 22 year review. IRCMJ. 2006;8(3):4-21. 